\title{
Structural conformation of lipids at the oil-water interface
}

\author{
Mario Campana ${ }^{\mathrm{a}}$, John R. P. Webster ${ }^{\mathrm{b}}$, M. Jayne Lawrence ${ }^{\mathrm{c}}$ and Ali Zarbakhsh ${ }^{{ }^{\mathrm{a}}}$ \\ ${ }^{a}$ School of Biological \& Chemical Sciences, Queen Mary, University of London, \\ Joseph Priestley Building, Mile End Road, London E1 4NS, UK. \\ ${ }^{\mathrm{b}}$ ISIS neutron facility, Science and Technology Facilities Council, Rutherford Appleton Laboratory, \\ Harwell Science and Innovation Campus, Didcot, OX11 0QX, UK. \\ ${ }^{\mathrm{c}}$ Institute of Pharmaceutical Science, King's College London, Franklin-Wilkins Building \\ 150 Stamford Street, London SE1 9NH, UK.
}

*Author to whom correspondence should be addressed. Email: a.zarbakhsh@qmul.ac.uk

\begin{abstract}
A neutron reflectivity study of the phospholipid, 1,2-distearoyl-sn-glycero-3-phosphocholine, at the hexadecane/water interface is reported as a function of spread amount. Two isotopic contrasts have been used to determine the structure of the phospholipid molecule in the buried interfacial region. The results indicate a roughened monolayer at low spread amounts of phospholipid at the oilwater interface. This monolayer is relatively broad to accommodate the coulombic charges on the head group. An increase in the spread amount of phospholipid results in combination of a monolayer plus micelle formation at the interface. There is a transition from a monolayer to a more complex monolayer and micelle conformation as the amount of spread phospholipid increases. The total layer thickness for these fits is about $70 \AA$, which is much larger than a fully extended DSPC molecule ( $30 \AA$ ). This is indicative of rough molecular packing at the oil-water interface. This roughened interface is suggested to be because of the solvation effect of the hydrocarbon tails and the resultant hydrophobic interactions, in addition to the accommodation of the coulombic interactions of the charges in the head groups.
\end{abstract}




\section{INTRODUCTION}

Monolayers composed of amphiphilic molecules, such as phospholipids, are widely regarded as model biomembranes for use in biophysical studies for the exploration of biomembrane properties and behaviour. Because of the presence of two long, fatty acid chains in a phospholipid molecule [1], such molecules are very sparingly soluble in water yet are considerably soluble in organic solvents, and thus structural studies of phospholipids at water-organic solvent interfaces are potentially a very important tool in understanding biomembrane structure and function. One area of special interest is the molecular conformation of the phospholipid head groups since these comprise the interaction site with the aqueous environment and other hydrophilic biomolecules such as solvated proteins [2,3].

Phospholipids such as dialkyl phosphocholine are zwitterionic in nature. The study of single alkyl chain zwitterionic surfactants at the air-water interface using deuterated and hydrogenous species has revealed that the hydrocarbon part of the adsorbed film forms a relatively thin and well packed layer with the alkyl chains being significantly tilted relative to the surface normal $[4,5]$. A similar tilt in the chain conformations has also been reported for monolayers prepared solely from dialkyl chain zwitterionic phospholipids $[6,7,8]$ and from mixtures of zwitterionic and anionic phospholipids [9] at the air-water interface. When zwitterionic surfactants are adsorbed at the oilwater interface, however, the adsorbed monolayer becomes much rougher and less organised because of the solvation conditions of the surfactant's tail groups [10]. Despite their very poorly solubility in water and alkanes, lipids have been reported to form micelles and unilamellar vescicles in both oil and water after sonication. Such systems have often been used for self assembly [11]. Lipids such as dipalmitoyl-phosphocholine (DPPC) can form stable Langmuir monolayers at the oil-water interface. Structural studies of DPPC adopting X-ray reflectivity have shown a well ordered monolayer at the hexadecane-water interface [12]. However, X-ray experiment reported was only performed at surface pressure $\gamma=10 \mathrm{mN} \mathrm{m}^{-1}$, and there is a lack of systematic studies of the effect of increasing surface coverage.

The contrast variation technique used in neutron reflectomtery provides a powerful tool in resolving conformation of molecules at the buried oil-water interface. However, until recently, it has not been possible routinely to resolve experimentally the structure of amphiphilic molecules located at such buried interfaces. The problem has been mainly due the lack of suitable methodology to successfully perform the experiment [13]. We have deployed our novel methodology for resolving the structure of amphiphilic phospholipid molecules at the interface between two immiscible liquids using neutron reflectometry for the first time. 
This methodology has enabled us to resolve the conformation of both the tail and the head groups of the lipid, 1,2-distearoyl-sn-glycero-3-phosphocholine (DSPC), for different spread amounts using neutron reflectometry.

In this paper we report the structural studies of monolayer prepared from 1,2-distearoyl-snglycero-3-phosphocholine at the oil-water interface for a series of spread amounts. The principal objectives were first to measure the adsorbed amount and to examine how the related interfacial phospholipid structure varies with concentration.

\section{EXPERIMENTAL SECTION}

\section{Materials}

1,2-distearoyl-sn-glycero-3-phosphocholine (DSPC), available as fully hydrogenous (h) and with the two stearoyl chains deuterated (d) forms, were obtained from Avanti Polar Lipids, Inc. Hexadecane-d34 was obtained from Cambridge Isotope Laboratories (> 98 atom D\%). Hydrogenous hexadecane was purchased from Aldrich and was purified by passing through an alumina column seven times. $\mathrm{D}_{2} \mathrm{O}$ was obtained from Fluorochem (>99 at. $\mathrm{D} \%$ ), and ultrapure $\mathrm{H}_{2} \mathrm{O}$ was produced using an Elgastat water purification unit. DSPC monolayers were spread on top of an aqueous subphase from a chloroform solution using a recently developed technique [14]. Details are given in the methods section. When chain deuterated d-DSPC was studied, the hexadecane oil and the aqueous sub-phase were both contrast matched to silicon $\left(\mathrm{Nb}=2.07 \times 10^{-6} \AA^{-2}\right)$ by the judicious mixing of appropriate amounts of hydrogenous and deuterated forms of hexadecane and water, respectively. A second contrast was also examined using h-DSPC lipid in which the oil phase was again contrast matched to the silicon and $\mathrm{D}_{2} \mathrm{O}$ was used as the aqueous sub-phase. In both contrasts the ionic strength of the aqueous phase, $I$, was zero. The oil-water interface was formed using existing methodology, namely the spin-freeze-thaw method [13].

\section{Methods}

Neutron reflectivity measurements were carried out at the ISIS Spallation Neutron Source, Rutherford Appleton Laboratory, Didcot, U.K. using the SURF reflectometer [15]. The neutron beam on this instrument is a polychromatic beam having a wavelength in the range 0.53 to $6.9 \AA$. The momentum transfer, $\mathrm{Q}$, is defined by $Q=(4 \pi \sin \theta) / \lambda$. The reflectivity profiles were measured at an incident angle of $1.5^{\circ}$ to provide the most suitable Q-range possible and the highest sensitivity to interfacial structure. The sample was under illuminated with resolution, $\delta \Theta / \Theta \sim 3.0 \%$. The attenuation of the neutron beam upon transmission through the oil layer was minimized by using a thin oil layer film created by spin-coating it onto a $100 \mathrm{~mm}$ diameter silicon block, which had been 
previously rendered hydrophobic by the coupling of chlorotrimethylsilane. The spun film of hexadecane oil was frozen in place and kept frozen for the cell-assembling procedure. In the current experiment, the water subphase was placed in the trough forming a meniscus, and its surface was cleaned by vacuum suction. Before assembly of the cell, the level of the water surface was lowered, by syringing through plug valves, until it was just lower than the O-ring seal. A known amount of DSPC was deposited on the bulk aqueous surface from a chloroform solution. After spreading, the solvent was allowed to evaporate ( 15 minutes) prior to the introduction of the oil phase. Once the sample chamber was deemed to be bubble-free, the oil film was allowed to melt. The linear adsorption coefficient, $\chi$, for the oil phases determined previously [16] was used in the data analysis. We have used our well established thick film approximation method [17] to calculate the total reflectivity from the Si-oil-water interface. The white beam nature of the neutron source used means the neutron transmission through the oil phase is wavelength-dependent hence all the data analysis are carried out as a function of wavelength but for reasons of clarity are all shown as a function of momentum transfer, Q. The data are all normalised to unit reflectivity in the normal way.

\section{RESULTS}

The spin coating procedure leads to a reproducible oil layer of $2.1 \mu \mathrm{m}$ thickness [16]. The oil layer thickness is relatively much thicker than the molecular dimensions of the lipid at the interface and the oil can be assumed to be bulk for the lipid. In order to deduce the full conformation of DSPC uniquely, two sets of contrasts were measured and the resulting reflectivity data fitted simultaneously to a consistent model. For the first contrast, a series of reflectivity profiles were measured as a function of spread amount of d-DSPC, with both the oil and water scattering length density $(\mathrm{Nb})$ being matched to that of the silicon. The normalised reflectivity profiles as a function scattering wave vector $\mathrm{Q}\left(\AA^{-1}\right)$ for the four lipid concentrations used are shown in Figure 1. Note that the reflectivity profiles obtained for when the d-DSPC concentrations were $7.47 \times 10^{-6}$ and $9.96 \times 10^{-6} \mathrm{~mol} \mathrm{~m}^{-2}$ overlay each other. The reflectivity from a bare oil-water interface was first measured as a reference bench mark and is also shown in Figure 1. The reflectivity data were first fitted to a single layer model of thickness $d=38 \pm 2 \AA$ as the first step in the data analysis. The one layer fit approach to the reflectivity data is an established technique [18] to estimate a nominal value for the adsorbed amount at a given interface. This standard approach is based on fitting the data to a single layer characterised only by a layer thickness with zero roughness and an $\mathrm{Nb}$ value. This approach is solely used to estimate the adsorbed amount and by no means aims to provide any structural details at a given interface. The fits are shown by the solid lines in Figure 1 and the fitted parameters are given in Table 1. As the data for the higher lipid concentrations of $9.96 \times 10^{-6}$ and $7.47 \times 10^{-6} \mathrm{~mol} \mathrm{~m}^{-2}$ were very similar (within error), one model could be used to represent both sets of data. The fitted layer 
thickness $(d)$ and the scattering length density were used to estimate the adsorbed amount $(I)$ of lipid and the area per lipid molecule $\left(A_{p m}\right)$ as explained below.

In order to ascertain the volume fraction of our system from the scattering length density profile, we need to make a simplifying assumption. This reasonable, simplifying assumption is that the scattering length density of the adsorbed lipid layer $\left(\mathrm{Nb}_{\text {layer }}\right)$ varies in a linear fashion with composition i.e. with the volume fraction of the lipid in the layer. As a consequence therefore, the volume fraction $(\Phi)$ profile of the adsorbed lipid layer can be related to the experimentally determined $\mathrm{Nb}_{\text {layer }}$ as:

$$
\Phi=\frac{N b_{\text {layer }}-N b_{b}}{m}
$$

where $\mathrm{Nb}_{\mathrm{b}}$ is the scattering length density of the bulk phase (in our case silicon, as both the oil and water are matched to silicon, $\left.\mathrm{Nb}_{\mathrm{b}}=2.07 \times 10^{-6} \AA^{-2}\right)$ and $m\left(m=\frac{\Delta y}{\Delta x}=\frac{N b_{\text {lipid }}-N b_{b}}{1}\right)$ is the gradient of the linear regression line in Figure 2. Using $\mathrm{Nb}_{\text {lipid }}\left(\mathrm{Nb}_{\text {lipid }}=4.78 \times 10^{-6} \AA^{-2}\right)$, the scattering length density of the lipid, equation (1) can be rewritten as:

$$
\begin{gathered}
\Phi=\frac{N b_{\text {layer }}-N b_{b}}{N b_{\text {lipid }}-N b_{b}} \\
\Gamma=\frac{d \rho}{M W} \times \frac{N b_{\text {layer }}-N b_{b}}{N b_{\text {lipid }}-N b_{b}}
\end{gathered}
$$

$\Gamma$ is expressed in units of mol m${ }^{-2}, d$ is the layer thickness (determined experimentally), $\rho$ is the lipid density and MW is the lipid molecular weight. By rearranging the Equation [18] $\Gamma=\frac{1}{N_{A} A_{p m}}$ the area per lipid molecule can also be calculated:

$$
A_{p m}=\frac{1}{N_{A} \Gamma}
$$

Where $\mathrm{N}_{\mathrm{A}}$ is Avogadro constant. The area per lipid molecule was calculated as a function of spread amount from the single layer fit to the reflectivity data for the first contrast, and it is shown in Figure 3.

For the second contrast, a series of reflectivity profiles were measured for the h-DSPC lipid as a function of spread amount, with the oil contrast matched to silicon and $\mathrm{D}_{2} \mathrm{O}$ as the aqueous sub-phase. The nature of model fitting of the neutron reflectivity data, necessitates the finding of the simplest model (i.e. the model with the minimum number of layers) to represent the data. We found that at the lowest spread amount $\left(1.87 \times 10^{-6} \mathrm{~mol} \mathrm{\textrm {m } ^ { - 2 }}\right)$ the reflectivity profiles for both contrasts could adequately be represented by a two layer model representing a roughened monolayer. Whereas, the reflectivity profiles obtained for the second contrast at higher spread amounts of lipid required a minimum three layers in order to obtain a reasonable representation of the data sets. This result suggests that there is a distinct change of conformation of the lipid molecules at the buried interface as 
the amount of spread lipid increases. The fits to the reflectivity profiles of the second contrast (hDSPC) are shown by the solid lines in Figure 4. This multilayer model could also be used to successfully represent the data obtained for first contrast using d-DSPC. The multilayer fits to the first contrast are shown in the insert of Figure 1. The fitted parameters obtained for both contrasts are given in Table 2. The interfacial roughness in modelling any reflectivity data could be set to zero; however a step-like interface is not a realistic representation of the actual interface and normally a Gaussian roughness is used to smooth the $\mathrm{Nb}$ changes moving from one layer to the next. The scattering length density profiles for these fits are shown in Figure 5. Each layer is assumed to be composed of oil, water and lipid. The $\mathrm{Nb}$ of the layer can be expressed as:

$$
N b_{\text {layer }}=N b_{\text {oil }} \phi_{\text {oil }}+N b_{\text {water }} \phi_{\text {water }}+N b_{\text {lipid }} \phi_{\text {lipid }}
$$

Where $\Phi_{i}$ is the volume fraction of the specie $i$ and the sum of the volume fractions for the three species is $1\left(\sum_{i} \phi_{i}=1\right)$.. The Nb profiles for both contrasts are then used, applying Equation 5, to estimate the volume fraction profiles for all the three components of the interface, namely the oil, lipid and water. The volume fraction profiles are shown in Figure 6.

The total layer thickness for these multilayer fits is about $70 \AA$, which is much larger than a fully extended DSPC molecule ( $30 \AA)$. This is indicative of rough molecular packing at the oilwater interface. Similar findings have been reported previously in the literature for surfactants at these buried interfaces $[10,16]$. This roughened interface is suggested to be because of the solvation effect of the hydrocarbon tails and the resultant hydrophobic interactions, in addition to the accommodation of the coulombic interactions of the charges in the head groups.

\section{DISCUSSION}

The scattering length density profiles obtained from the model fittings of all the reported data are shown in Figure 5. The profiles for the higher spread amounts of h-DSPC with the $\mathrm{D}_{2} \mathrm{O}$ forming the aqueous sub-phase are shown in Figure 5(c). Regions (i) and (ii) of this profile correspond, respectively, to the head group region and the tail group of the primary monolayer. The decrease in the $\mathrm{Nb}$ in both regions as the spread amount increases indicates a combination of an increase in the lipid content coupled with an exclusion of $\mathrm{D}_{2} \mathrm{O}$ from the layer. This is probably a consequence of a higher packing density of the lipid monolayer in this region. On the contrary, the $\mathrm{Nb}$ of region (iii) of the profile, in the vicinity of the oil phase, increases with increased spread amount, suggesting the formation of (reversed) micelles. As more (reversed) micelles are formed in this region the water incorporated in this layer also increases. This increase could be because of the contribution of the hydration shell of the head groups. The micelle observed on the oil side of the interface could originate from spontaneous emulsification process. Spontaneous formation of water in oil micelle has 
been reported at the oil/water/lipid interface [19]. These micelles near the interface could exchange with other micelles in the bulk oil, however there is only sensitive to changes at the interface and very limited sensitivity to relatively small changes in the $\mathrm{Nb}$ of the bulk oil phase. There is an overall increase in the adsorbed amount in all three regions and this is also confirmed by the scattering length density profiles for the first contrast, shown in Figure 5 (a). Similar trend in the scattering length density profile is also observed for the lowest spread amount (Figure 5 (b) and 5 (d)) again both these two sets of contrasts are fitted simultaneously to the same model.

It is important to note that the reflectivity profiles (Figure 4) can also be satisfactory be modelled by relaxing the layer roughness (Figure 5(c) and (d) and fitting the data to smooth (diffuse) scattering length density profiles. These are shown in Figure 7. The rise (broad peak) in these profiles on the oil side of the interface could suggest the aggregate formation, packing of the head group with some D2O hydration head groups on the oil side of the interface.

The overall results are shown schematically in Figure 8 . The reflectivity data at low spread amount $\left(1.87 \times 10^{-6} \mathrm{~mol} \mathrm{~m}^{-2}\right)$ indicate a monolayer conformation of the lipid molecules at the oilwater interface. This monolayer is represented by two layers (Figure $5 \mathrm{~b}$ and $5 \mathrm{~d}$ ). The data further suggest this monolayer is rough with a staggered conformation to accommodate the coulombic interactions between the charges in the DSPC head groups. The first layer in contact with water is relatively well defined, mainly composed of DSPC head groups and water. The second layer is a mixture of lipid, hexadecane and water. The thickness of the second layer is $55 \AA$, which is more than twice the fully extended stearoyl chain; hence some hydrated lipid head groups must also be included in the composition of this layer. The layer appears to be disordered with a significant amount of water penetration. This water inclusion in the hydrophobic side of the interface has been already observed at the oil water interface [20] as well as for phospholipid at the air-water interface [8]. This can be attributed to an increase in interfacial roughness in the presence of surface active species.

In contrast 2, with $\mathrm{D}_{2} \mathrm{O}$ forming the aqueous phase, very little contrast exists between the head group and tail group hence less sensitivity to structural details obtained. The interface could be arbitrarily divided into more layers to demonstrate some structural order. However the increasing number of layers would drastically increase the number of fitting parameters, thus compromising the uniqueness of the final model. We believe a simple two layer model is a more realistic representation of the system given the experimental sensitivity. This type of molecular arrangement for molecules with charges in the head groups has been observed for a zwitterionic single chain C16 surfactant and has been reported in the literature [10]. As the spread amount of the lipids from chloroform increases a different conformation of lipids at the oil-water interface is observed and the reflectivity profiles can't be represented by a two layer model. The scattering length density (SLD) profiles suggest that as a 
small volume of lipid solution is spread on the water surface, a monolayer is formed at the interface as was implied from the lowest spread amount data. As more and more of the solution is spread subsequently on the top of this monolayer, the lipid molecules come into contact with a more hydrophobic environment composing of the lipid tail groups of the already formed monolayer. The spread lipids are now exposed to very little water and as a result there is a change in conformation of the newly spread lipids. Hence we move from a roughened monolayer to a monolayer plus lipid (water in oil) micelles. These micelles reside on the oil side of the interface in the vicinity of the monolayer.

The $\mathrm{Nb}$ profiles for the two contrasts (Figure 5) were used to calculate, using equation (5), the volume fraction $(\Phi)$ for the individual components (DSPC, water and hexadecane). This is a very simple estimation of the composition of each region of the interfacial layer. The volume fraction profiles for two spread amount values are shown in Figure 6. These volume fraction profiles suggest that the most of the lipids reside primarily on the aqueous side of the interface with a different degree of water penetration through the interface. However any oil in the interfacial region is confined to the layer adjacent to the bulk oil phase. The values for the area per molecule at the oil-water interface are shown in Figure 3. The values obtained are significantly higher than those reported in the literature [8] for the same DSPC lipid at the air-water interface ( 49 to $44 \AA^{2}$ with surface pressure increasing from 20 to $50 \mathrm{mN} / \mathrm{m}^{-1}$ ). The values are also higher than those reported for dipalmitoyl-phosphocholine (DPPC) at the air-water interface [21]. This is not surprising given the lipid layer at the oil-water are formed by spreading from a volatile solvent. This is because we cannot compress this insoluble monolayer at the oil-water interface with our current experimental setup. In addition the formation of micelles implies the depletion of materials from the interface. The area per molecule plot (Figure 3) shows a plateau region for the spread amount above $7.47 \times 10^{-6} \mathrm{~mol} \mathrm{~m}^{-2}$ indicating a possible saturation at the interface with the current experimental procedure. However given the lack of sensitivity of neutron reflectivity to the small changes in the bulk concentration we are not able to speculate with regard to where exactly the excess material reside.

\section{CONCLUSION}

The neutron reflectivity data using two contrasts have shown the monolayer formation for the DSPC lipid at a lowest amount of spread lipid at the oil-water interface. We have chosen the simplest layer structure to represent our data given the analysis of neutron reflectivity data is based purely on a model fits to the data. However this doesn't exclude the possibility of more complicated structures particularly for the lowest spread amount. The thickness of the monolayer is relatively high (about twice the molecular length) to accommodate the coulombic charges on the head group. An increase in the amount of spread lipid results in the formation of a monolayer plus possible micelle at the 
interface. It has been reported, when lipids are adsorbed at the oil-water interface from bulk phases, they have the tendency to self assemble into bilayers separated by thin water layers [19]. Events such as thermally-induced waves or the application of electric fields across the interface destabilise the multilayer structure. Defects are then generated in the bilayers which eventually lead to their disintegration and dispersion in the oil phase as water-in-oil emulsions or reversed micelles. Both spherical and rod-like lecithin micelles have been reported in non polar solvents, rod-like micelles being more stable at higher concentration [20]. The core of the micelles is very hydrophilic and is subject to significant hydration. It is believed that water transfers into the non polar solvent through hydration of the adsorbed lipid multilayer [21], triggering a series of phase transitions that, depending on the solvent, may lead to a marked increase in viscosity of the hydrophobic phase [22-24]. More recently, the spontaneous emulsification at a lipid-stabilised oil-water interface was reported [25] and was also attributed to the hydration of the adsorbed lipid multilayer.

The results confirm that spreading molecules at high concentration from a volatile solvent onto a limited surface may not be ideal. It seems there is a transition from a monolayer to a more complex monolayer and micelle conformation as the spread amount increases. At the air-water interface one would normally prepare the monolayer using a Langmuir trough, by spreading on a large area and then compressing the layer using a moveable barrier. This is not currently possible for the oil-water interface experiments using neutron technique because of the drastic attenuation of the neutron beam through the bulk upper oil phase. We have now designed a new trough with a focusing trumpet for the neutron beam and sets of experimental protocols to use a Langmuir-type trough for these experiments in the future. This new trough enables the molecular conformation using a bulk oil to be resolved at these buried interfaces in addition to enabling the compression of the spread monolayer. This experimental set up will allow us to determine whether the formation of micelles is a consequence of the way in which the lipid film was prepared or it is due to the consequence of the presence of oil for which it has a certain affinity.

\section{AUTHOR INFORMATION}

Corresponding Author

E-mail: a.zarbakhsh@qmul.ac.uk

\section{ACKNOWLEDGMENT}

The authors wish to thank the STFC, UK, for granting direct-access beam-time on SURF (ISIS) for this work and for part funding of a PhD studentship. 


\section{REFERENCES}

[1] Cantor, R. C. and Schimmel, P. R. Biophysical Chemistry; The Conformation of Biological Macromolecules. Vol. 1. W.

H. Freeman and Company, SanFrancisco, 1980.

[2] Möhwald, H. Ann. Rev. Phys. Chem. 1990, 41, 441.

[3] Naumann, C.; Dietrich, C.; Lu, J. R.; Thomas, R. K.; Rennie, A. R.; Penfold, J.; Bayerl, T. M. Langmuir 1994, $10,1919$.

[4] Yaseen M, Lu, J. R.; Webster J. R. P.; Penfold, J. Langmuir, 2006, 22 (13), 5825

[5] Hines, J. D.; Garrett, P. R.; Rennie, A. R.; Thomas, R. K.; Penfold, J. J. Phys. Chem. 1997, B101, 7121.

[6] Vankin D., Kjaer K, Als-Nielsen J., Lösche M., Biophys. J., 1991, 59, 1325.

[71 Brumm T., Naumann C., Sackmann E., Rennie A. R., Thomas R. K., Kanellas D., Penfold J., Bayerl T. M., Eur Biophys. J. 1994, 23, 289.

[8] Hollinshead C. M., Harvey R. D., Barlow D. J., Webster J. R. P., Hughes A. V., Weston A. Lawrence M. J., Langmuir, 2009, 25, 4070.

[9] Bayerl, T. M., Thomas R. K., Penfold J., Rennie A., Sackmann E., Biophys. J., 1990, 57, 1095.

[10] Zarbakhsh, A, Querol A, Bowers J. Yaseen M., Lu J. R. and Webster, J. R.P. Langmuir 2005, 21 (25), 11704.

[11] Johnson S., Bayerl T. M., McDermott D. C., Adam G. W., Rennie A. R., Thomas R. K., Sackmann E., Biophys J, 1991, 59, 289

[12] Fradin C., Luzet D., Braslau A., Alba M., Muller F., Daillant J., Langmuir, 1998, 14, 7327

[13] Zarbakhsh, A, Bowers, J. and Webster, J. R. P., M. Sci. Technol, 1999, 10, 738

[14] Zarbakhsh A, Webster JR, Wojciechowski K; Langmuir, 2009, 25 (19), 11569

[15] Penfold, J.; Richardson, R. M.; Zarbakhsh, A.; Webster, J. R. P.; Bucknall, D. G.; Rennie, A. R.; Jones, R. A. L.; Cosgrove, T.; Thomas, R. K.; Higgins, J. S.; Fletcher, P. D. I.; Dickinson, E.; Roser, S. J.; Mclure, I. A.; Hillman, A. R.;

Richards, R. W.; Staples, E. J.; Burgess, A. N.; Simister, E. A.; White, J. W. J. Chem. Soc., Faraday Trans, 1997, 93, 3899

[16] Zarbakhsh, A, Querol, A., Bowers, J., Webster, J. R.P., Faraday Disc. 2004, 129, 155

[17] Zarbakhsh, A, Bowers, J. and Webster, J.R.P. Langmuir. 2005, 21 (25), 11596

[18] Lu J.R., Thomas R.K., Penfold J., Adv. Colloid Interface Sci., 2000, 84, 143

[19] Pautot S., Frisken B. J., Cheng J. X., Xie X. S., Weitz D. A., Langmuir, 2003, 19, 10281

[20] Zarbakhsh A., Webster J. R. P., Eames J., Langmuir, 2009, 25, 3953

[21] Kundu, S.; Colloid surfaces A, 2010, 368, 31

[22] Scartazzini R., Luisi P. L., J Phys Chem, 1988, 92, 829.

[23] Schurtenberger P., Scartazzini R., Magid L. J., Leser M. E., Luisi P. L., J phys Chem, 1990, 94, 3695.

[24] Shchipunov Y. A., Shumilina E. V., Mat Sci Eng C, 1995, 3, 43.

[25] Pautot S., Frisken B. J., Cheng J. X., Xie S., Weitz D. A., Langmuir, 2003, 19, 10281. 


\section{Figure Captions}

Figure 1. Reflectivity spectra $\mathrm{R}(\mathrm{Q})$ from the system $\mathrm{Si}$-hexadecane contrast matched $\mathrm{Si}(\mathrm{Nb}=2.07 \times$ $\left.10^{-6} \AA^{-2}\right)$ - water contrast matched $\mathrm{Si}\left(\mathrm{Nb}=2.07 \times 10^{-6} \AA^{-2}\right)$ for a series of d-DSPC lipid spread amount and fitted using a single layer fits. Insert are fits obtained using multilayer model using log (Reflectivity). The solid lines are fits to the data.

Figure 2. Relationship between scattering length density $(\mathrm{Nb})$ profile and volume fraction profile.

Figure 3. Area per molecule determined from a one layer fit to the data versus the spread amount.

Figure 4. Reflectivity spectra $\mathrm{R}(\mathrm{Q})$ from the system $\mathrm{Si}$-hexadecane contrast matched $\mathrm{Si}(\mathrm{Nb}=2.07 \times$ $\left.10^{-6} \AA^{-2}\right) / \mathrm{D}_{2} \mathrm{O}\left(\mathrm{Nb}=6.35 \times 10^{-6} \AA^{-2}\right)$ for a series of h-DSPC lipid spread amount. The solid lines are fits to the data. The profiles are shifted by a factor $\times 10$ for the purpose of clarity.

Figure 5. Scattering length density $(\mathrm{Nb})$ profiles for calculated reflectivity profiles shown in Figures 1 (insert) and 4.

Figure 6. Volume fraction profiles of the lipid distribution for two spread amounts $\left(7.47 \times 10^{-6}\right.$ and $1.87 \times 10^{-6} \mathrm{~mol} \mathrm{~m}^{-2}$ ) deduced from the two contrasts. The short-dash represents the lipid volume fraction profile, solid line is the water and the long-dash is the oil.

Figure 7. A continuously changing Scattering length density $(\mathrm{Nb})$ profiles for calculated reflectivity profiles shown in Figure 4 is shown here. These scattering length density profiles equally represent the data similar to a fixed layer roughness model of Figure 5 (d) and (c).

Figure 8. Schematic representation of the conformation of DSPC at low (left) and high (right) spread amounts. A change in conformation is observed as the spread amount increases. 


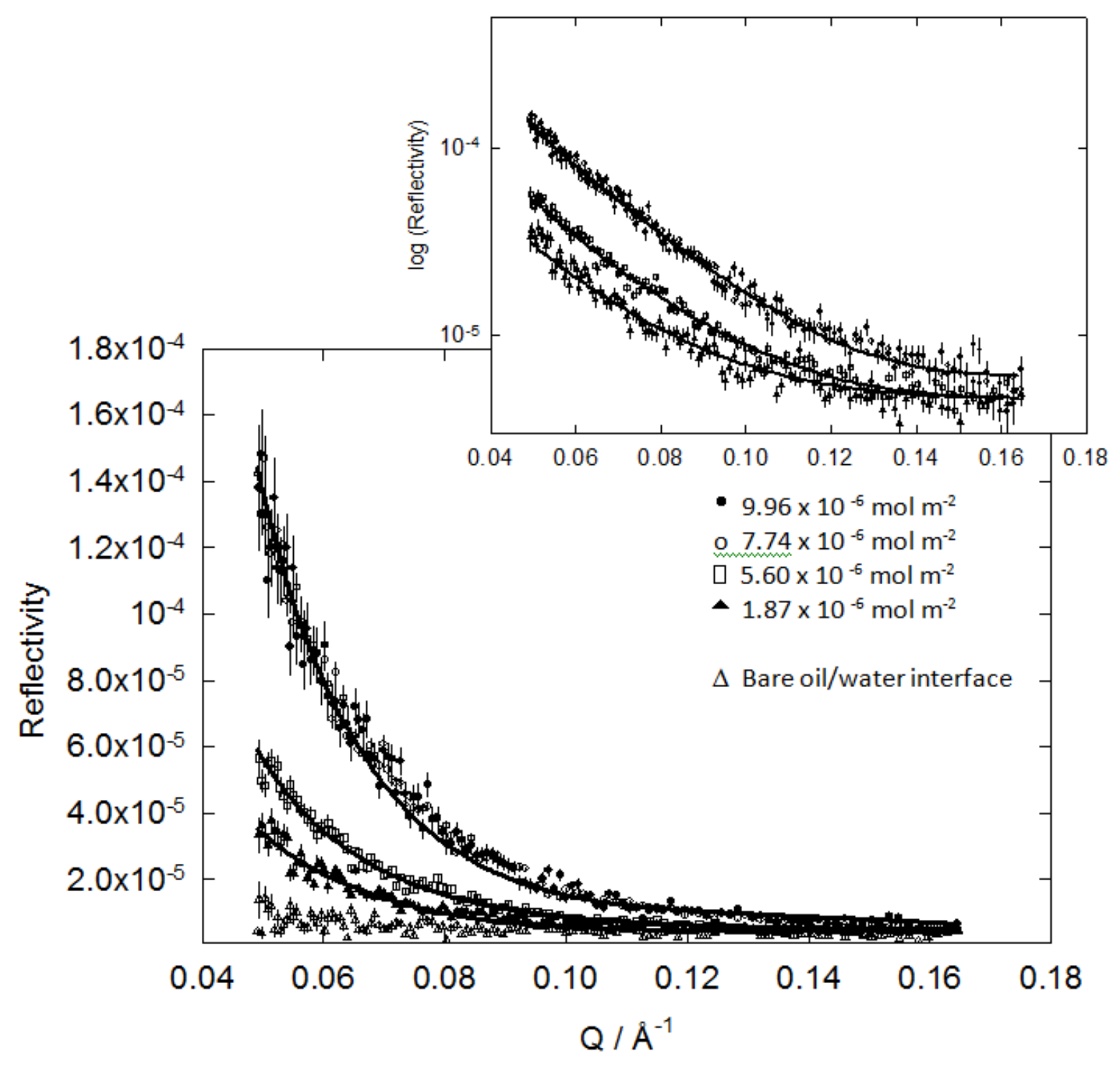

Figure 1. 


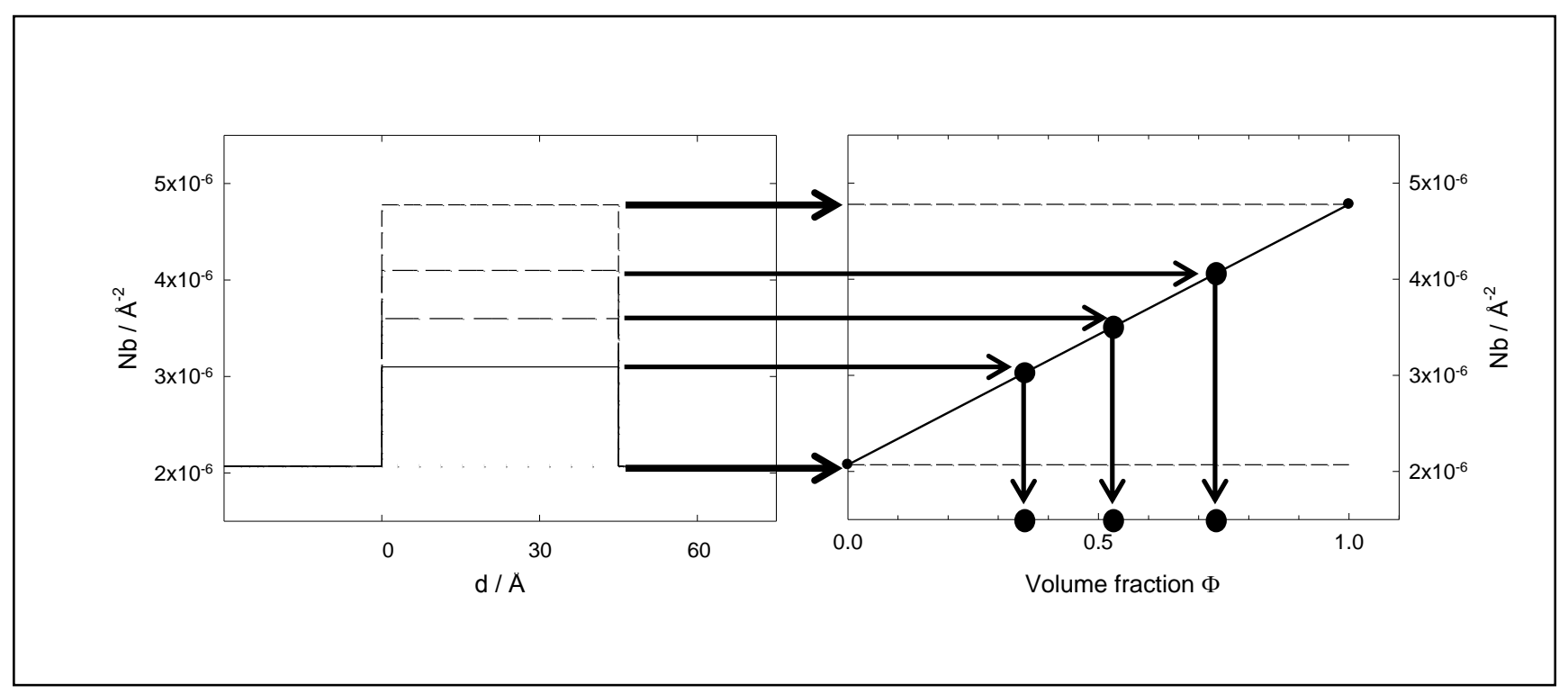

Figure 2. 


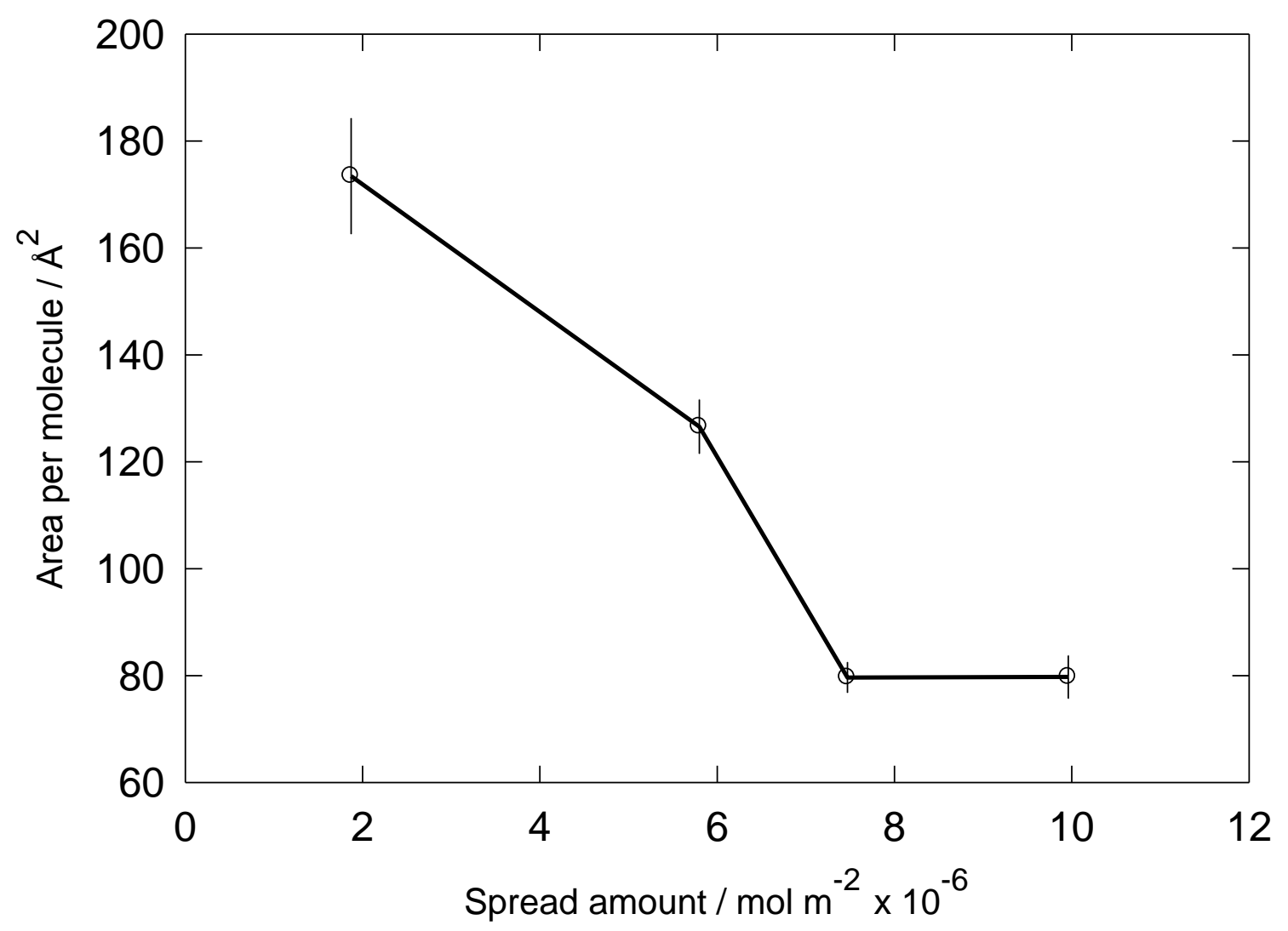

Figure 3. 


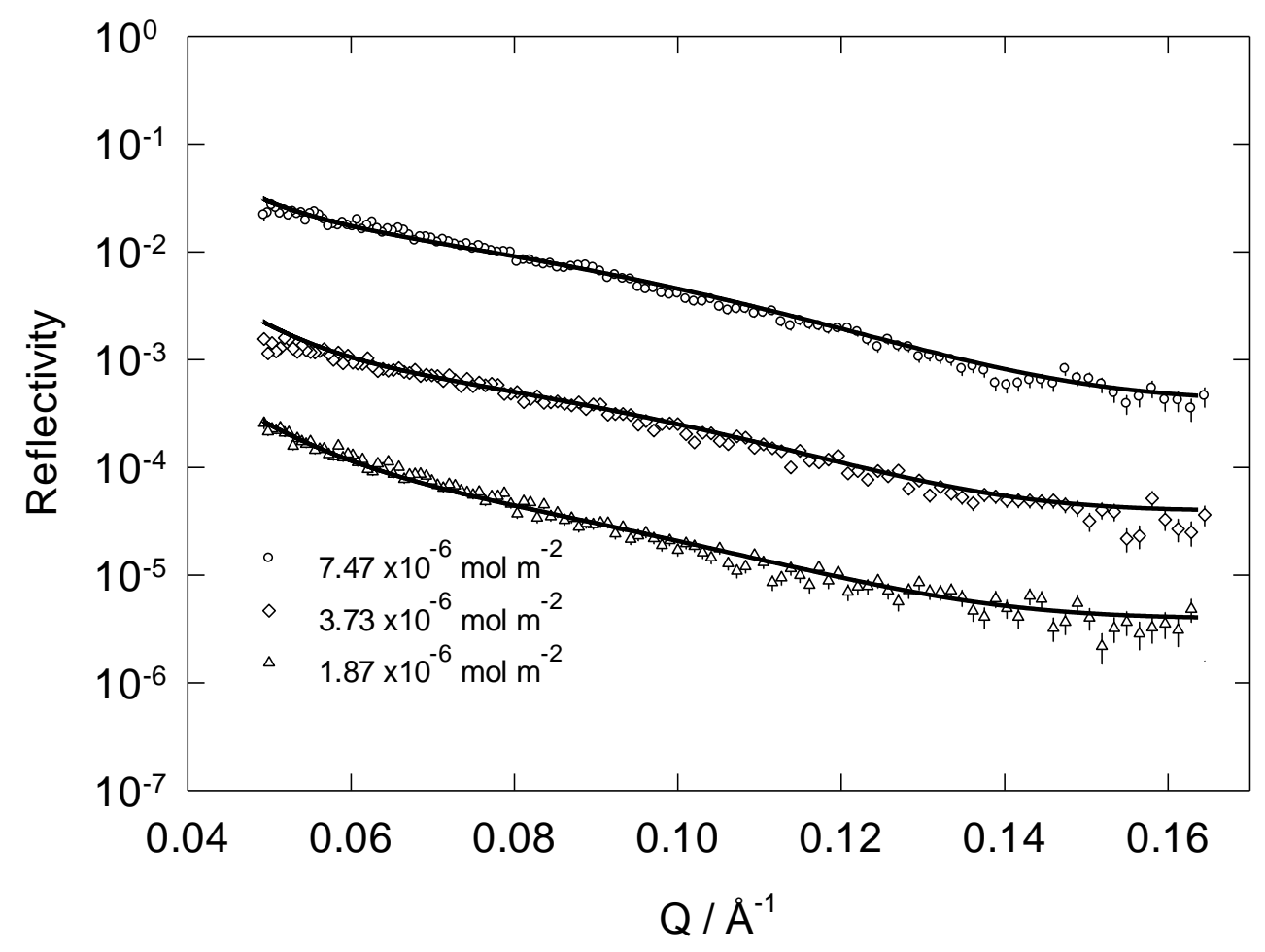

Figure 4 


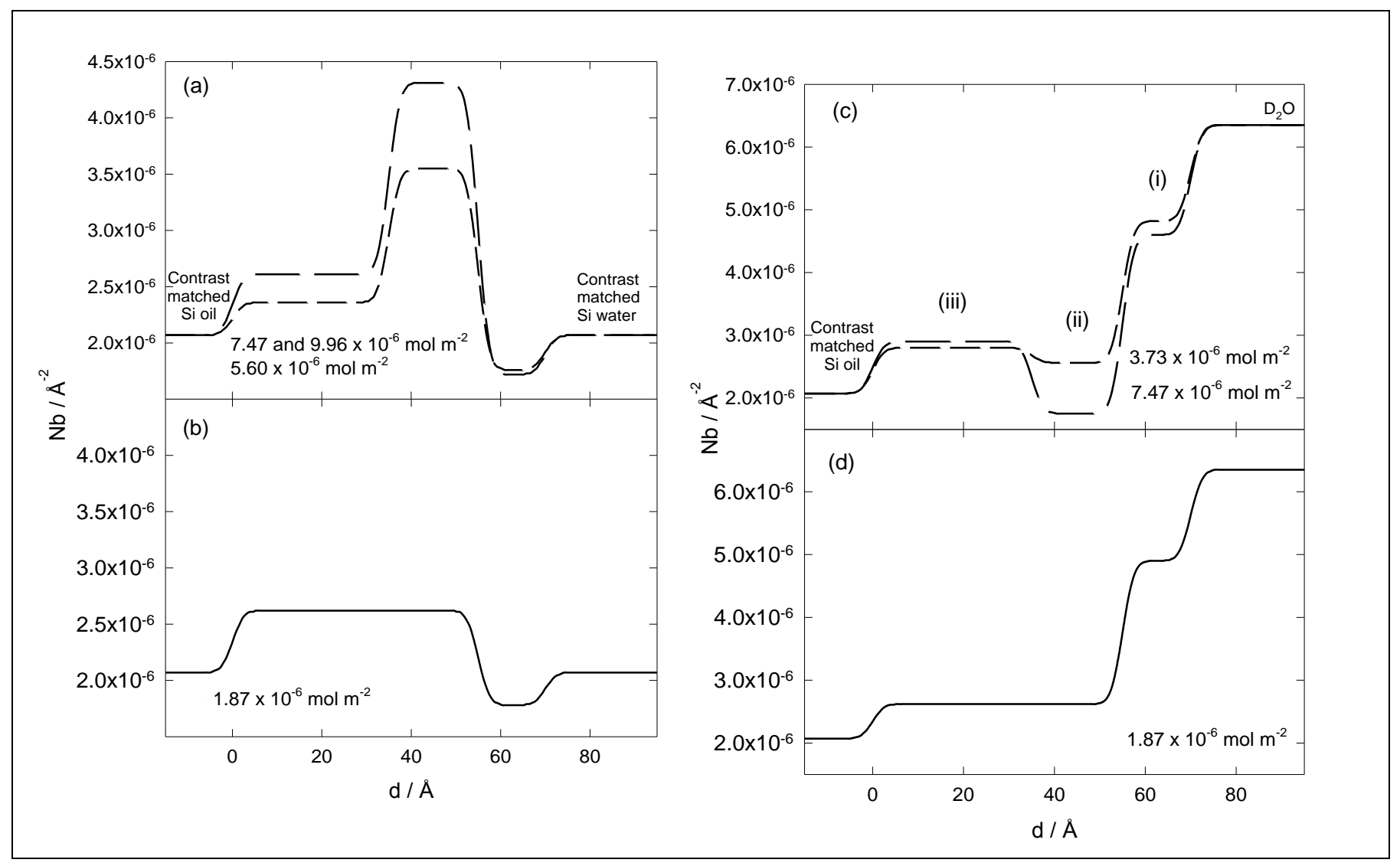

Figure 5 


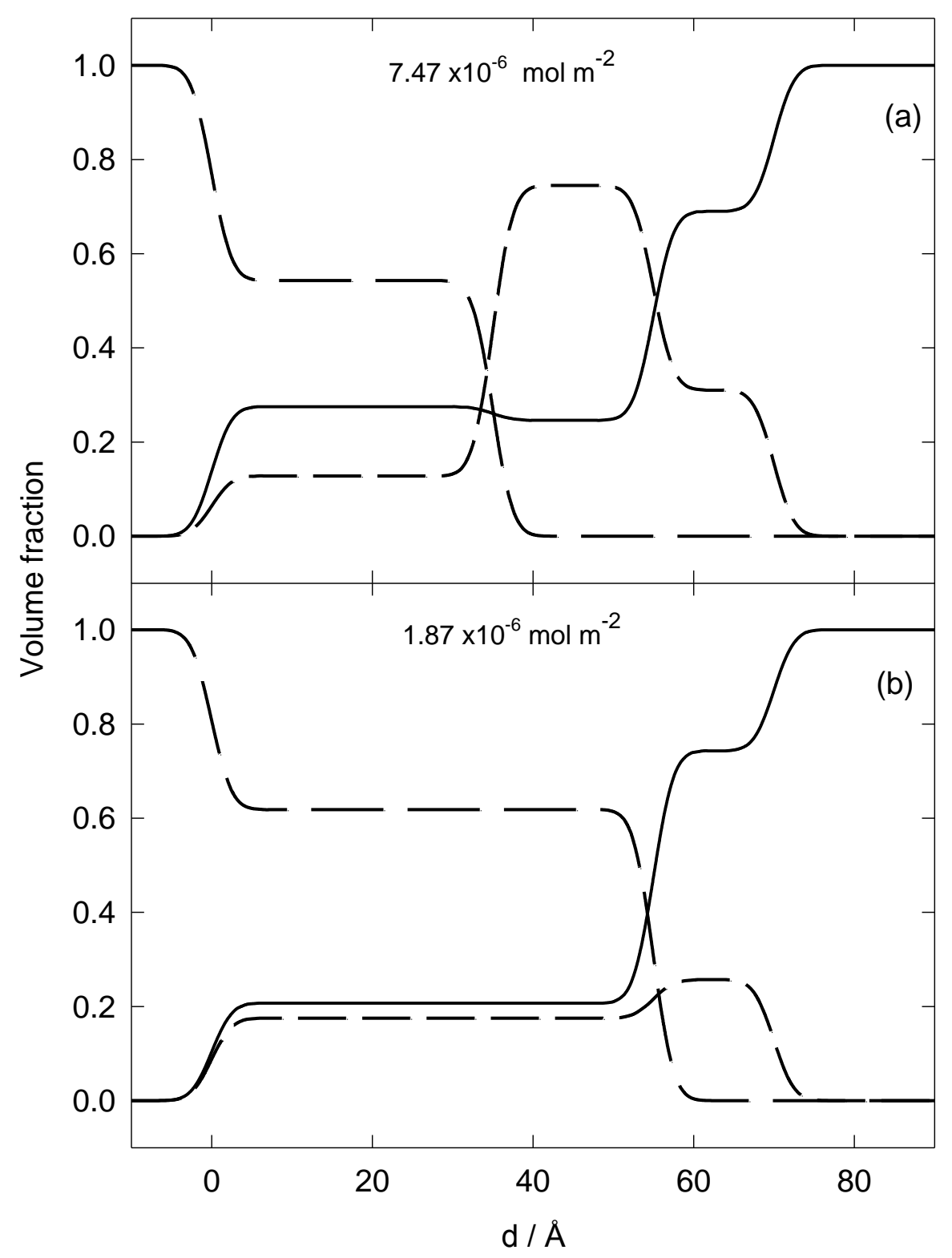

Figure 6. 


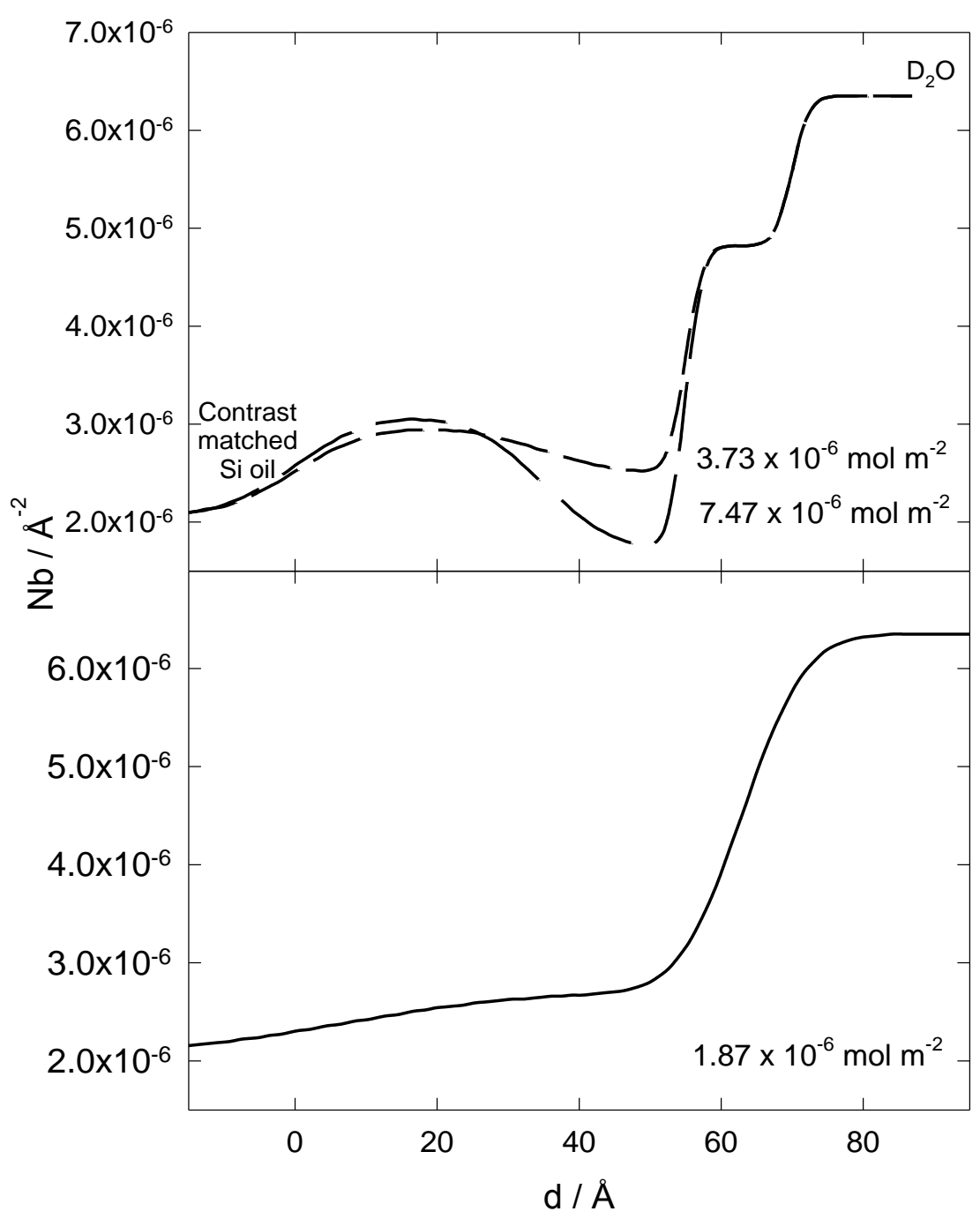

Figure 7. 


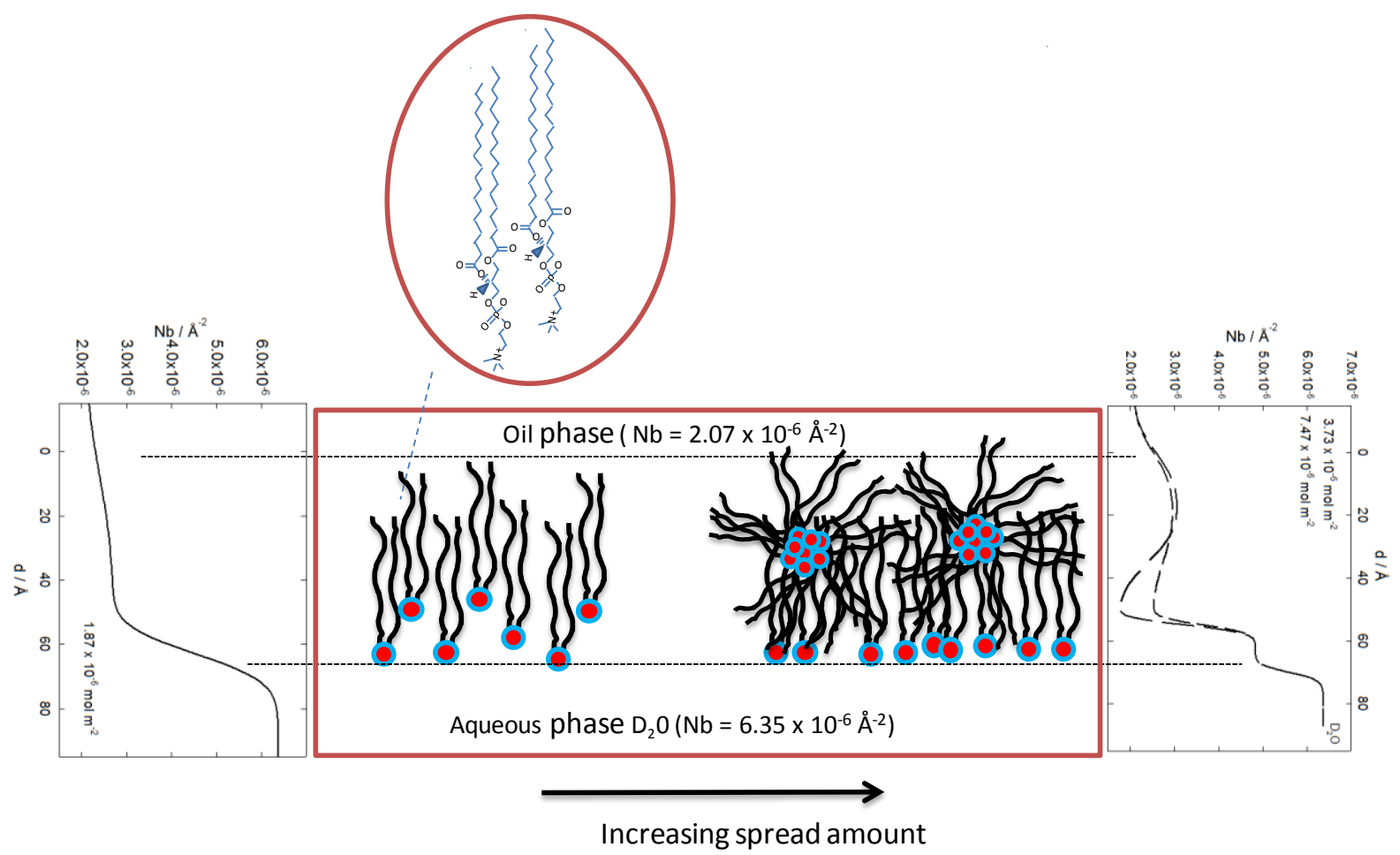

Figure 8. 


\begin{tabular}{|l|l|l|l|l|}
\hline Spread amount $(\Gamma) \mathrm{d}$-DSPC $\times 10^{-6} / \mathrm{mol} \mathrm{m}^{-2}$ & 1.87 & 5.60 & 7.47 & 9.96 \\
\hline $\mathrm{Nb} \times 10^{-6} / \AA^{-2}$ & 2.67 & 2.91 & 3.40 & 3.40 \\
\hline
\end{tabular}

Table 1. Parameters used for one layer fit (Figure 1) for contrast 1 with a layer thickness of $38 \pm 2 \AA$

Contrast 1: Contrast match Si oil / d-DSPC/Contrast match Si water, lowest spread amount

\begin{tabular}{|c|c|c|}
\hline $\mathrm{d}$-DSPC mol m & -2 \\
\hline $\mathrm{d} / \AA( \pm 1 \AA)$ & $\mathrm{Nb} \times 10^{-6} / \AA^{-2}$ & roughness $/ \AA$ \\
\hline 55.0 & 2.59 & 2.0 \\
\hline 15.0 & 1.78 & 2.0 \\
\hline
\end{tabular}

Contrast 1: Contrast match Si oil / d-DSPC/Contrast match Si water, higher spread amount

\begin{tabular}{|c|c|c|c|c|}
\hline d-DSPC $\mathrm{mol} \mathrm{m}^{-2}$ & $9.96 \times 10^{-6}$ & $7.47 \times 10^{-6}$ & $5.60 \times 10^{-6}$ & \\
\hline $\mathrm{d} / \AA( \pm 1 \AA)$ & \multicolumn{3}{|c|}{$\mathrm{Nb} \times 10^{-6} / \AA^{-2}$} & roughness / $\AA$ \\
\hline 35.0 & 2.61 & 2.61 & 2.36 & 2.0 \\
\hline 20.0 & 4.31 & 4.31 & 3.55 & 2.0 \\
\hline 15.0 & 1.72 & 1.72 & 1.76 & 2.0 \\
\hline
\end{tabular}

Contrast 2: Contrast match Si oil / h-DSPC/ $\mathrm{D}_{2} \mathrm{O}$, lowest spread amount

\begin{tabular}{|c|c|c|}
\hline $\mathrm{h}-\mathrm{DSPC} \mathrm{mol} \mathrm{m}^{-2}$ & $1.87 \times 10^{-6}$ & \\
\hline $\mathrm{d} / \AA( \pm 1 \AA)$ & $\mathrm{Nb} \times 10^{-6} / \AA^{-2}$ & roughness $/ \AA$ \\
\hline 55.0 & 2.62 & 2.0 \\
\hline 15.0 & 4.90 & 2.0 \\
\hline
\end{tabular}

Contrast 2: Contrast match Si oil / h-DSPC/ $D_{2} \mathrm{O}$, higher spread mount

\begin{tabular}{|c|c|c|c|}
\hline h-DSPC mol m-2 & $7.47 \times 10^{-6}$ & $3.73 \times 10^{-6}$ & \\
\hline $\mathrm{d} / \AA( \pm 1 \AA)$ & \multicolumn{2}{|c|}{$\mathrm{Nb} \times 10^{-6} / \AA^{-2}$} & roughness $/ \AA$ \\
\hline 35.0 & 2.90 & 2.80 & 2.0 \\
\hline 20.0 & 1.75 & 2.56 & 2.0 \\
\hline 15.0 & 4.60 & 4.82 & 2.0 \\
\hline
\end{tabular}

Table 2. Parameters used for multilayer fits to both contrast. The calculated fits are shown by the solid lines in Figure 1 (insert) and Figure 3. 


\section{Table of contents entry}

\section{Structural conformation of lipids at the hydrophilic-hydrophobic interface}

Mario Campana ${ }^{1}$, John R. P. Webster ${ }^{2}$, M. Jayne Lawrence ${ }^{3}$ and Ali Zarbakhsh ${ }^{1^{*}}$

${ }^{1}$ School of Biological \& Chemical Sciences, Queen Mary, University of London, Joseph Priestley Building, Mile End Road, London E1 4NS, UK.

${ }^{2}$ ISIS neutron facility, Science and Technology Facilities Council, Rutherford Appleton Laboratory, Harwell Science and Innovation Campus, Didcot, OX11 0QX, UK.

${ }^{3}$ Institute of Pharmaceutical Science, King's College London, Franklin-Wilkins Building 150 Stamford Street, London SE1 9NH, UK.

*Author to whom correspondence should be addressed. Email: a.zarbakhsh@qmul.ac.uk

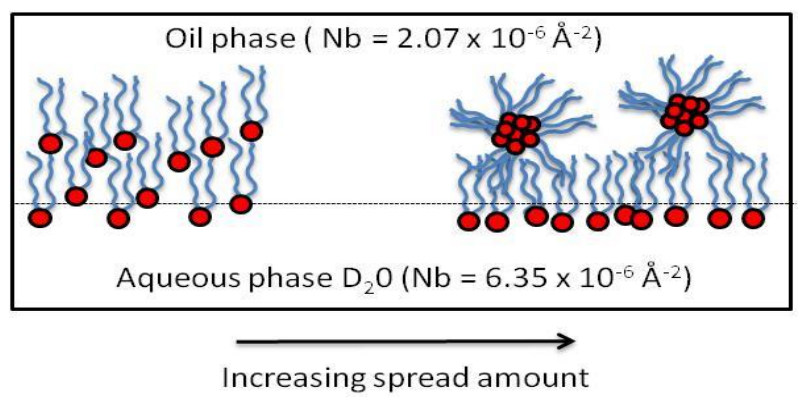

Schematic representation of the conformation of DSPC at low (left) and high (right) spread amounts. A change in conformation is observed as the spread amount increases. 